\title{
EVALUACIÓN DE MORTEROS A COMPRESIÓN APROVECHANDO EL RESIDUO AGROINDUSTRIAL DE CRATAEGUS MEXICANA (TEJOCOTE) EN MORTEROS
}

\author{
M. C. GARCÍA ${ }^{1,2}$, H. L. CHAVÉZ ${ }^{1,2}$, L. COLÍN ${ }^{1,2}$, I. TRUJILLO $^{1}$, F. CRUZ ${ }^{1}$ \\ ${ }^{1}$ Facultad de Ingeniería Civil, Laboratorio de Materiales, Universidad Michoacana de San Nicolas de Hidalgo. \\ ${ }^{2}$ Maestría en Ciencias en Ingeniería Ambiental, Universidad Michoacana de San Nicolas de Hidalgo.
}

\section{RESUMEN}

La investigación se enfoca en determinar si los residuos agroindustriales como el hueso de tejocote son óptimos para la elaboración de un mortero base cemento sin modificar sus aplicaciones ingenieriles. El Crataegus mexicana (Tejocote) es de origen mexicano. Se realizaron pruebas físicas y mecánicas a las muestras. Una de las pruebas que se realizó a cubos de morteros con dimensiones de $5 \times 5 \mathrm{~cm}$ es la prueba a compresión. Se elaboraron 3 tipos de muestras (testigo, adición y sustitución) y se probaron 5 especímenes para cada muestra, las edades de prueba fueron 14,28, 45 y 90 días. Se uso arena que obtenida del banco de materiales llamado "Joyitas" ubicado en la carretera Morelia-Jiquilpan en el estado de Michoacán; el agua que se utilizó cumple las especificaciones de la norma NMX-C122ONNCCCE.,2004; el aditivo empleado fue Crisotan ${ }^{\circledR}$ R-5, aditivo de la gama de los plastificantes (fluidificantes); y por último se adicionó (5\%) o sustituyó (5\%) el hueso de tejocote que fue caracterizado como si fuese una arena

\section{ABSTRACT}

The research focuses on determining if agroindustrial residues such as tejocote bone are optimal for the production of a cement-based mortar without modifying its engineering applications. The Mexican Crataegus (Tejocote) is of Mexican origin. Physical and mechanical tests were performed on the samples. One of the tests that was performed on mortar cubes with dimensions of $5 \times 5 \mathrm{~cm}$ is the compression test. Three types of samples (control, addition and substitution) were prepared and tested for the ages of 14, 28, 45 and 90 days. The additive used was Crisotan ${ }^{\circledR}$ R-5, (fluidiser); and finally $(5 \%)$ was added or replaced $(5 \%)$ the tejocote bone that was characterized as if it were sand.

\section{RESUMO}

A pesquisa se concentra em determinar se os resíduos agroindustriais, como o osso tejocoto, são ótimos para a produção de argamassa à base de cimento, sem modificar suas aplicações de engenharia. $\mathrm{O}$ crataegus mexicano (Tejocote) é de origem mexicana. Testes físicos e mecânicos foram realizados nas amostras. Um dos testes realizados em cubos de argamassa com dimensões de $5 \times 5 \mathrm{~cm}$ é o teste de compressão. Três tipos de amostras (controle, adição e substituição) foram preparados e testados para as idades de 14, 28, 45 e 90 dias. O aditivo utilizado foi o Crisotan ${ }^{\circledR}$ R-5 (fluidizante); e finalmente (5\%) foi adicionado ou substituído (5\%) o osso tejocote que foi caracterizado como se fosse areia. 


\section{INTRODUCCIÓN}

El concreto es el material más usado en la construcción. Con el aumento de la industrialización y la urbanización, una gran cantidad de recursos naturales son requeridos para la elaboración de concreto en todo el mundo. Para eliminar o minimizar el impacto ambiental negativo sobre la industria del concreto y promover un ambiente sustentable de la industria, el uso de los desechos industriales como materiales para la elaboración de concreto es considerado como una solución alternativa para la prevención de un déficit de materia prima.

La disponibilidad de desechos agroindustriales hace de ellos una alternativa adecuada y confiable como agregados en el concreto.

Unos de los objetivos es promocionar la metodología y el uso de estos desechos en la elaboración de mortero y que permanezcan las mismas aplicaciones ingenieriles.

El área de infraestructura y construcciones verdes se ha ido expandiendo en la última década. En general, el actual sistema de clasificación de construcciones verdes (Green Building Rating, GBR) evalúa la sustentabilidad de las construcciones en varias categorías, en las cuales los materiales de construcción están presentes en la mayoría de las categorías. Uno de los problemas es la emisión de $\mathrm{CO}_{2}$ durante la producción de cemento Portland, así como una significativa cantidad de energía, agua, agregados y rellenos usados en la producción de concreto, así como los desechos de la construcción por demolición de estructuras de concreto, hace que el cemento como material de construcción luzca menos compatible con los requerimientos ambientales que una industria de construcción sustentable moderna requiere.

La utilización de desechos industriales y agrícolas puede ser un parteaguas en la transformación de esta industria haciéndola más amigable con el medio ambiente y sustentable. Los materiales residuales, tales como la ceniza volante, humo de sílice, residuos de la planta de explotación de hornos de escoria, concreto reciclado, vidrio reciclado, llantas recicladas, y reciclado de plásticos, han sido usados exitosamente en el concreto por décadas (Meyer C., 2009). Además, estudios recientes han demostrado el éxito en el uso de los desechos sólidos agrícolas como agregados en el concreto estructural y no estructural.

\section{DESARROLLO EXPERIMENTAL}

\subsection{Agregados}

Para el desarrollo de esta investigación el agregado fino utilizado en los morteros fue obtenido del banco de materiales llamado "Joyitas" que está ubicado en el kilómetro 14+500 de la la carretera Morelia-Jiquilpan entrada a San Bernabé en el estado de Michoacán, el tipo de propiedad es particular, el material obtenido es volcánico y el tratamiento que se le da a dicho material es trituración parcial y cribado; no requiere explosivos para la obtención del material y económicamente hablando el material es aceptable.

El agregado volcánico del banco "La Unión", fue tomado de un depósito de material y llevado al sitio de estudio para realizarle las pruebas correspondientes de laboratorio. Una vez colocado en un compartimento de materiales se tamizó por la malla No. 4 para llegar al tamaño máximo de agregado fino.

Los usos probables que se le dan a ese material son como material de revestimiento sub - base, base, y en la elaboración de concreto hidráulico. 


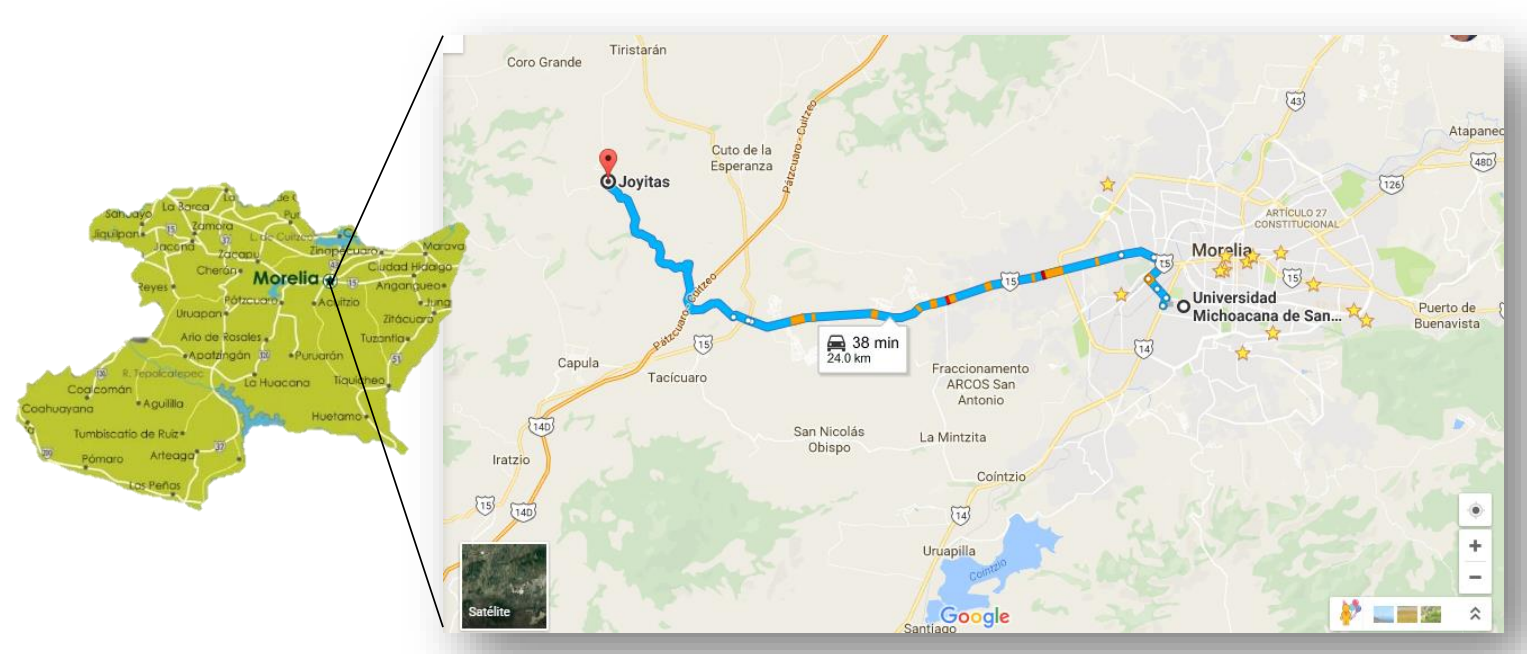

Figura 1. Ubicación del banco la unión con referencia Morelia, Mich

Para conocer las propiedades y características de este agregado se le efectuaron diferentes pruebas como:

- Cuarteo en arenas (NMX-C-170-ONNCCE, 1997)

- Humedad actual (NMX - C - 166 (NMX-C-166-ONNCCE., 2006)

- Humedad superficial y humedad de absorción (NMX-C-165-ONNCCE., 2014)

- Determinación de la masa Volumétrica seca y suelta (NMX-C-073-ONNCCE., 2004)

- Determinación de la masa volumétrica seca y varillada (NMX-C-073-ONNCCE., 2004)

- Granulometría en arenas (NMX-C-077-ONNCCE., 1997), (NMX-C-251-ONNCCE., 1997) (NMX-C-111-ONNCCE, 2014

\subsection{Agua}

Prácticamente cualquier agua natural que sea potable y no presente fuerte sabor u olor se puede usar como agua de mezcla para la preparación del concreto. Sin embargo, también se pueden emplear en concreto algunas aguas que no se consideran potables. Se puede utilizar satisfactoriamente el agua para la preparación del concreto con menos de 2000 partes por millón (ppm) de sólidos disueltos. El agua que contiene más de 2000 ppm de sólidos disueltos se debe analizar para verificar su efecto sobre la resistencia y el tiempo de fraguado.

El agua utilizada en la investigación para realizar las mezclas y el curado de los morteros cumple con la norma NMX-C-122-ONNCCE., 2004. El agua se utilizó tal y como llega al laboratorio, esto con el fin de usarla lo más apegado a las condiciones reales de obra. Se sabe que en la construcción no toman en cuenta la calidad del agua, la utilizan tal y como se la suministran y no importa si está contaminada.

\subsection{Aditivo}

El aditivo que se utilizó para la elaboración de mezclas es uno distribuido principalmente para la construcción, su nombre comercial es Crisotan ${ }^{\circledR}$ R-5, aditivo de la gama de los plastificantes (fluidificantes), su presentación es en polvo con un color café oscuro. El Crisotan® R-5 es un agento NO TÓXICO, que no provoca espuma. Y dado que es un producto sintético, presenta propiedades uniformes de un lote a otro, a diferencia de los lignosulfonatos.

Su estabilidad térmica se garantiza hasta los $150^{\circ} \mathrm{C}$. Su temperatura de descomposición es a los $380^{\circ} \mathrm{C}$. En dosificaciones normales, el Crisotan ${ }^{\circledR}$ R-5 no afecta al calor de hidratación del concreto. No contribuye a la corrosión del acero, ni al crecimiento de microorganismos. 
La dosificación del Crisotan® R-5 se basa en el contenido de la mezcla de concreto. En la mayoría de los casos se puede obtener una reducción de agua con un $0.5 \%$ de Crisotan ${ }^{\circledR}$ R-5 (en base al ingrediente activo), o $1.66 \%$ para el Crisotan® R-5 líquido.

Para obtener las máximas ventajas del Crisotan ${ }^{\circledR}$ R-5, la mezcla de concreto debe ser rediseñada y probada. Un revenimiento bajo, puede ser corregido con una mayor disificación del Crisotan® R-5 en vez de agregar agua.

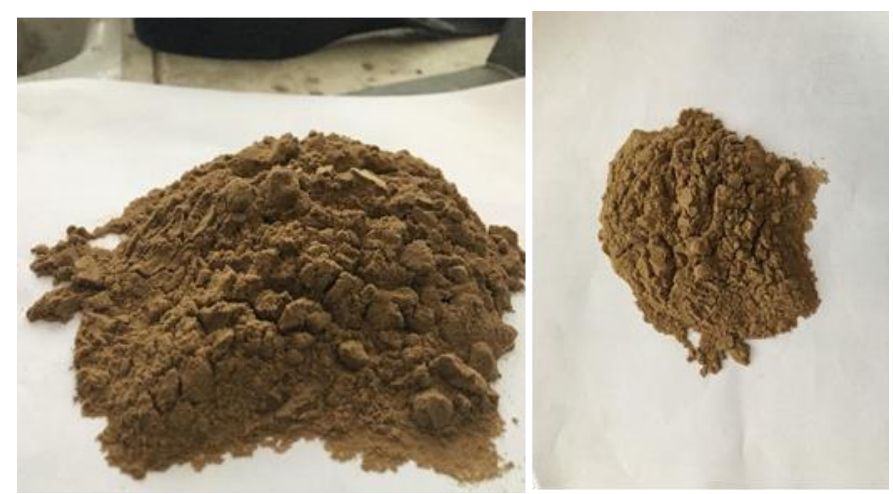

Figura 2. Fluidificante Crisotan R-5 (Vaca Arciga, L. A., 2017)

\subsection{Crataegus mexicana (Tejocote)}

El tejocote pertenece a la familia de las Rosáceas, al género Crataegus, el cual agrupa alrededor de 150 especies en todo el mundo, de las cuales 95 de ellas se encuentran en el continente americano, siendo 13 de ellas originarias de México (Phipps, 1997). La importancia del tejocote radica en su potencial uso como fruta fresca, portainjerto de otros frutales, forraje, fuente de pectina, ornamental y Medicinal (Nieto-Angel, 1991), y su uso en la preparación de licores tradicionales y conservas. El nombre tejocote, es el más extendido en México, es de origen náhuatl, de las raíces "tetl” piedra y xocotl (agrio o ácido). Es un árbol o arbusto caducifolio, de 4 a $10 \mathrm{~m}$ de altura y a menudo provisto de espinas. Su copa es ovoide extendida, con sombra densa; mientras que sus hojas son simples, alternas, romboides-elípticas u ovado lanceoladas, agudas, margen aserrado, en forma irregular, haz verde oscuro y glabro; envés más pálido y a veces pubescente. Su tronco es recto con ramas rígidas con espinas y su corteza es de color gris rojiza y se desprende en tiras.

Cuando el árbol de Crataegus mexicana alcanza la edad de maduración, presentan flores en forma de umbelas terminales con 2 a 6 flores; con pétalos blancos, ovado-orbicularas, de 7 a $10 \mathrm{~mm}$ de largo y florecen desde el mes de enero hasta abril.

$\mathrm{Su}$ fructificación empieza en primavera, pero maduran hasta noviembre o diciembre. Sus frutos son semejantes a una pequeña manzana, amarilla-anaranjada, de 1 a $2 \mathrm{~cm}$ de diámetro. Las semillas que son el objeto de investigación son ortodoxas, de color café, lisas y están rodeadas por un endocarpo o hueso leñoso duro, teniendo en cuenta que por cada kilogramo de tejocote se obtienen aproximadamente 5000 semillas. Este tipo de semillas puede almacenarse con contenidos de humedad de 6 a $7 \%$ y temperaturas $\leq 0^{\circ} \mathrm{C}$; tales condiciones permiten mantener la viabilidad por varios años. Generalmente las semillas ortodoxas presentan algún tipo de reposos, en el caso de esta especie las semillas presentan latencia primaria de tipo mecánica, en condiciones naturales la germinación de las semillas es favorecida por el fuego.

El material en estudio (Huesos de tejocote) fue caracterizado como si fuese una arena. Ya que este será utilizado como sustitución o adición de este agregado.

\subsection{Elaboración de especímenes}

Para la elaboración de los cubos de mortero en sus diferentes sustituciones, se utilizaron moldes de acero inoxidable y bronce que cumplen con las dimensiones especificadas por la norma correspondiente (NMX-C-159-ONNCCE, 2004) para la fabricación de cubos. Una vez realizado el llenado de cubos se 
procedió a curar los cubos de mortero (el curado para todos los especímenes como son: cubos, cilindros, prismas y briquetas fue el mismo) el curado que se dio a los especímenes fue por inmersión en agua hasta el día de la prueba (ASTM-C-515, 2003).

\subsection{Resistencia a la Compresión}

La prueba se realizado de acuerdo a la norma mexicana (NMX-C-403-ONNCCE., 1999). En el caso de esta investigación, cuya medida se da en ensayes de especímenes cúbicos elaborados, curados y probados en las condiciones estándar especificadas, generalmente expresada en $\mathrm{kg} / \mathrm{cm}^{2}$.

Realizar la prueba para conocer la resistencia a compresión, es lo más común de todas las pruebas sobre concreto o mortero endurecido, esto porque es de las características deseables del concreto o mortero, aunque no todas, están relacionadas cualitativamente con su resistencia (Neville \& Brooks, 2010).

Se utilizaron 5 especímenes cúbicos de $5 \times 5 \mathrm{~cm}$ por cada edad de prueba, es decir 15 cubos en total para las edades de 14, 28 y 45 días; y para cada una de las mezclas elaboradas.

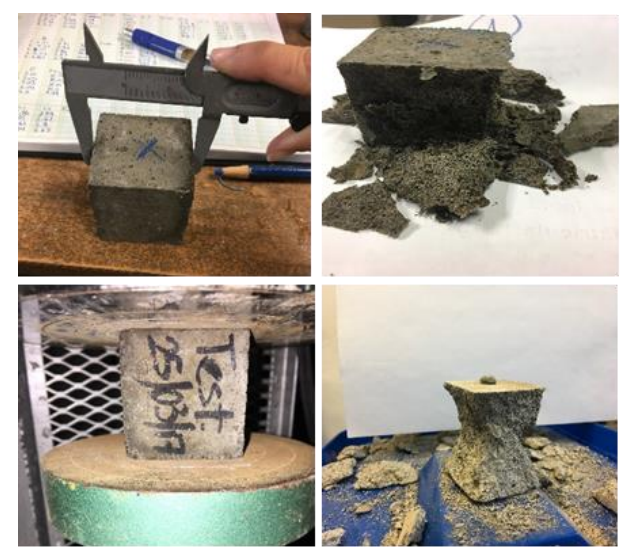

Figura 2. Prueba de compresión simple en cubos. (Vaca Arciga, L. A., 2017)

Cálculos.

$$
R=\frac{W}{A}
$$

Donde:

$\mathrm{R}=$ Resistencia a la compresión, en $\mathrm{kgf} / \mathrm{cm}^{2}$.

$\mathrm{W}=$ Carga de ruptura de la muestra, en $\mathrm{kg}$.

$\mathrm{A}=$ Área de la muestra, en $\mathrm{cm}^{2}$.

Tabla de registro.

Tabla 1 Tabla de registro para resistencia compresión simple

\begin{tabular}{c|c|c|c|c|c|}
\multicolumn{1}{c}{$\begin{array}{c}\text { No. de } \\
\text { espécimen }\end{array}$} & $\begin{array}{c}\text { Lado 1 } \\
\mathrm{cm}\end{array}$ & \multicolumn{1}{c}{$\begin{array}{c}\text { Lado 2 } \\
\mathrm{cm}\end{array}$} & \multicolumn{1}{c}{$\begin{array}{c}\text { Área } \\
\mathrm{cm}^{2}\end{array}$} & $\begin{array}{c}\text { Carga } \\
\mathrm{kg}\end{array}$ & $\begin{array}{c}\text { Resistencia } \\
\mathrm{Kgf} / \mathrm{cm}^{2}\end{array}$ \\
\hline & & & & & \\
\cline { 4 - 7 } & & & & & \\
\end{tabular}

Referencia.

(NMX-C-083-ONNCCE, 2014). 


\section{RESULTADOS}

La resistencia a la compresión simple de las diferentes mezclas respecto al mortero control, alcanza valores de hasta $23.60 \mathrm{MPa}$ (mezcla de HT -5\%) a los 45 días de edad, quedando una diferencia de 3 MPa por debajo de la mezcla testigo (Test.).

En la edad de 45 días el resultado de la mezcla de HT -5\% (23.60 MPa) demostró un comportamiento mejor que el de la mezcla de $\mathrm{HT}+5 \%$ (19.85 MPa); este comportamiento podría atribuirse a un mejor acomodo de partículas, o a la disminución del agregado fino sustituido por HT.

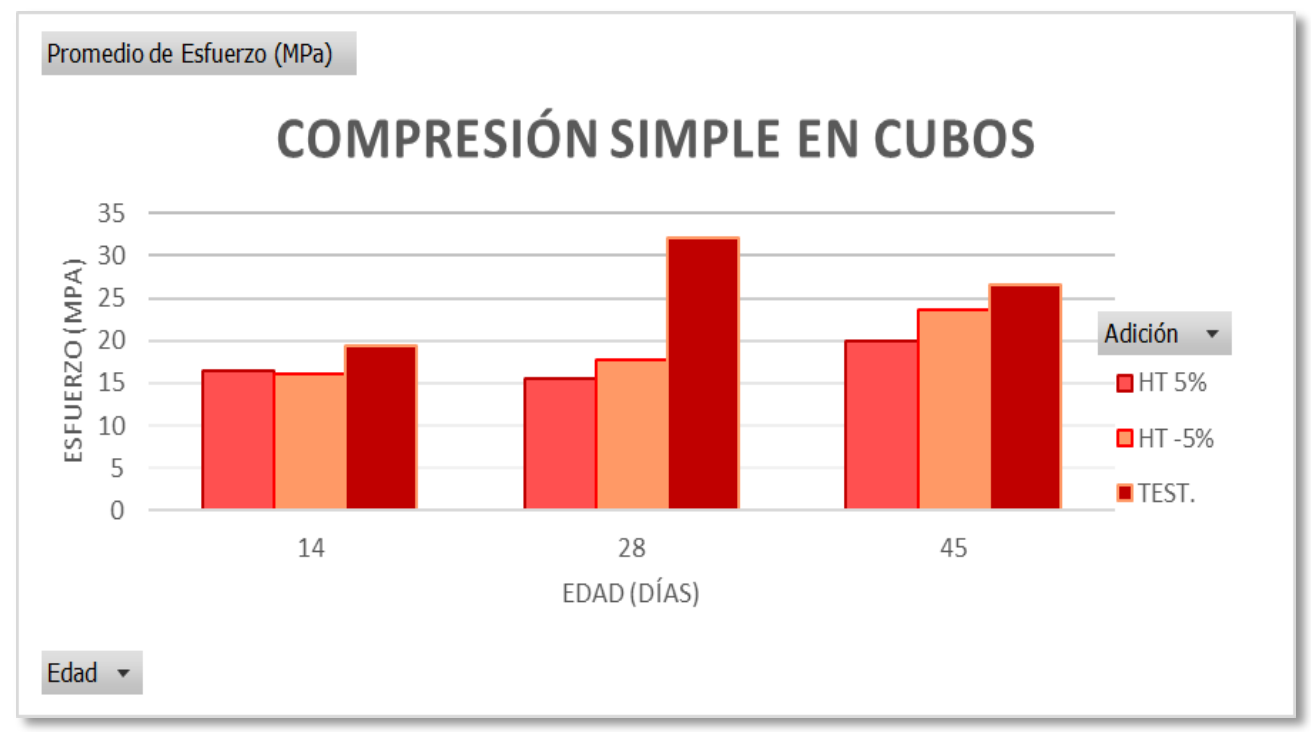

Figura 3 Resultados de compresión simple en cubos, a edades tempranas.

\section{CONCLUSIONES}

El uso de desechos agrícolas dentro de la industria de la construcción tiene un gran potencial para reducir de alguna manera la contaminación. Ya existe un número de desechos agrícolas que se han utilizado como un remplazamiento total o parcial del agregado. En esta investigación se utilizó el desecho agroindustrial del tejocote, siendo un desecho de la región. Sin embargo, una gran necesidad es el de estudiar, encontrar y caracterizar otros materiales de desecho. Por lo que en esta investigación nos centramos en conocer las propiedades físico-mecánicas de este desecho.

Las mezclas que se llevaron a cabo para la elaboración de morteros fueron: Una adición del 5\% de semillas de tejocote $(\mathrm{HT}+5)$ y una sustitución del 5\% del mismo material (HT -5\%), ambas mezclas modificando al agregado, y se elaboró una mezcla control o testigo con la cual se compararon los resultados.

De acuerdo a los criterios de evaluación de la (RED DURAR (Durabilidad de la armadura), 2000) las tres mezclas desde los 14 hasta los 45 días se clasifican con una calidad de mortero alta, de acuerdo a los resultados obtenidos de la prueba de velocidad de pulso ultrasónico.

La mezcla con mejores resultados es el control (test). De las mezclas con desechos, la mezcla HT $+5 \%$ obtuvo un mejor comportamiento en lo referente a las pruebas no destructivas, concluyendo que la densificación de estos especímenes es buena. Por otro lado, la mezcla de HT-5\% obtuvo mejores resultados en la cuestión de las pruebas destructivas, sin superar al mortero testigo. 


\section{AGRADECIMIENTOS}

Al Laboratorio de Materiales "Ing. Luis Silva Ruelas" de la Universidad Michoacana de San Nicolás de Hidalgo, departamento de Resistencia de Materiales, Corrosión y a toda persona que lo constituye, por brindar las instalaciones, material, equipo y apoyo necesario para desarrollar la presente investigación.

\section{REFERENCIAS}

AASHTO-T-106. (2002). Standar Test Method for Compressive Strength of Hydraulic Cement Mortars (Using 2-in, or [50 mm] Cube Specimens). EE.UU.

Alonso. (2017).

Ashurst, J. (1983). Mortars, plasters and renders in conservation.

ASTM C494/C494M -08a. (s.f.). Especificación Normalizada de Aditivos Químicos para Concreto. EE. UU.: ASTM.

ASTM-C-109 M, I. (2002). Standard Test Method for Compressive Strength of Hydraulic Cement Mortars (Using 2-in. or [50-mm] Cube Specimens). EE.UU.

ASTM-C-109-M. (2002). Standar Test Method for Compressive Strength of Hydraulic Cement Mortars (Using 2-in, or [50 mm] Cube Specimens). EE.UU.

ASTM-C-128. (2004). Standard Test Method for Relative Density (Specific Gravity) and Absorption of Fine Aggregate. E.E.U.

ASTM-C142/C142M. (2010). tandard Test Method for Clay Lumps and Friable Particles in Aggregates. EE.UU.: ASTM.

ASTM-C-151-00. (2014). Método estandar de ensayo para expansión en autoclave del cemento portland. EE.UU.: ASTM.

ASTM-C-348. (1997). Standard Test Method for Flexural Strength of Hydraulic - Cement Mortars. EE.UU.

ASTM-C-515. (2003). Standard Specification for Mixing Rooms, Moist Cabinets, Moist Rooms, and Water Storage Tanks Used in the Testing of Hydraulic Cements and Concretes. EE.UU.

ASTM-C-597. (2009). Standard Test Method For Pulse Velocity Through Concrete. Estados Unidos.

ASTM-G57. (2012). Standard Test Method for Field Measurement of Soil Resistivity Using the Wenner Four Electrode Method. EE.UU.

Batis, A. M.-Y. (1999). Árboles y Arbustos Nativos Potencialmente Valiosos para la Restauración Ecológica y Reforestación. México D.F.

Cemento, C. N. (2016). http://www.canacem.org.mx.

Charles, J. H. (2001). Handbook of Nondestructive Evaluation. Estados Unidos: McGrae-Hill.

Christianson, S. d. (s.f.). Crisotan ${ }^{\circledR}$ R-5, Dispersantes para concretos. CDMX.

Correa, R. S. (1984). Diseño de Mezclas de Mortero. Colombia Bogotá: Facultad de Ingeniería, Universidad Nacional de Colombia, Bogotá.

Definición de agua. (2017). Definición.de. Obtenido de http://definicion.de/agua/

Deuteronomio 5-27.2. (s.f.). La Biblia, Deuteronomio 5-27.2.

Frizot, M. (1975). Mortiers et enduits peints antiques-étude technique et archéologique.

Furlan. (1975). Les mortiers anciens. Histoire et essais d'analyse scientifique. Revue suisse d'Art et d’Archéologie. 32:1-14.

Ghorab, H., Ragai, J., \& Antar, A. (1986). urfare and bulk properties of ancient egyptian mortars. Part I: X-ray diffraction studies. 16:813-822.

Instituto del Concreto. (1997). Manual Tecnología y Propiedades. colombia: Asociación Colombiana de productores de concreto.

Kosmatka, S. H. (2004). Diseño y control de mezlas de concreto. Chicago, EE.UU: Portland Cement Association, PCA.

Kosmatka, S. H., Kerkhoff, B., Panarese, W. C., \& Tanesi, J. (2004). Diseño y control de mezlas de concreto. Chicago, EE.UU: Portland Cement Association, PCA.

L. RIVERA, I. G. (s.f.). CONCRETO SIMPLE-TECNOLOGÍA DEL CONCRETO. Colombia: civilgeeks.com. La Flor de Morelia. (04 de 07 de 2017). La Flor de Morelia. Obtenido de http://laflordemorelia.com/ 
Malinowski. (1982). Ancient mortars and concretes: Aspects of their durability. Histoire of Technology 7th Annual Volume. UK: 89-101.

Malinowski, G. Y. (1991). Prehistory of Concrete. Concrete. 32:1-14.

Martinet. (1992). Caractérisation des mortiers pharaoniques du temple d'Amon à Karnak. . 181:39-45.

Martínez Molina, W., Navarro Sánchez, L. M., \& Espinoza Mandujano, A. (2011). Tiempos de fraguado utilizando las agujas de Gillmore. En Análisis de Materiales. Morelia, Michoacán, México: Facultad de Ingeniría Civil.

Mendoza Anaya, D., Brito Benitez, E., \& Arenas Alatorre, J. (2004). La Ciencia de Materiales y su Impacto a la Arqueología. México: Academia Mexicana de Ciencia de Materiales.

Meyer C. (2009). The greening of the concrete industry. Cement Concrete Composite. EE.UU.: 31:601-5. PAG. Neville, A., \& Brooks, J. (2010). Concrete Technology. England: ISBN 978-0-273-73219-8.

Nieto-Angel, R. B. (1991). El tejocote (Crataegus spp.) en México: Avances en el Estudio de los Recursos Fitogenéticos en México. CDMC.

NMX-C-021-ONNCCE. (2015). INDUSTRIA DE LA CONSTRUCCIÓN-CEMENTO PARA ALBAÑILERÍA (MORTERO)-ESPECIFICACIONES Y MÉTODOS DE ENSAYO. CDMX: ONNCCE.

NMX-C-030-ONNCCE. (2004). Industria de la construcción - Agregados - Muestreo. CDMX: ONNCCE.

NMX-C-057-ONNCCE. (2015). Industria de la Construcción-Cementantes Hidráulicos-Determinación de la Consistencia Normal. CDMX: ONNCCE.

NMX-C-061-ONNCCE. (2015). Industria de la Construcción-Cemento Hidráulico-Determinación de la resistencia a la compresión de cementantes Hidráulicos. México: Organismo Nacional de Normalización y Certificación de la Construcción y la Edificación, S.C.

NMX-C-061-ONNCCE. (2015). Industria de la Construcción - Cemento Hidráulico - Determinación de la Resistencia a la Compresión de Cementantes Hidráulicos. CDMX: ONNCCE.

NMX-C-062-ONNCCE. (2010). Industria de la construcción - Cementos hidráulicos - Determinación de la sanidad de cementantes hidráulicos. CDMX: ONNCCE.

NMX-C-071-ONNCCE. (2004). Industria de la construcción - Agregados - Determinación de terrones de arcilla y partículas deleznables. CDMX: ONNCCE.

NMX-C-073-ONNCCE. (2014). Industria de la Construcción. Agregados. Masa Volumétrica. Método de prueba. México: Organismo Nacional de Normalización y Certificación de la Construcción y Edificación, S.C. NMX-C-073-ONNCCE. (2004). Industria de la Construcción - Agregados - Masa Volumétrica - Método de Prueba. CDMX: ONNCCE.

NMX-C-075-ONNCCE. (2006). Industria de la construcción - Agregados - Determinación de la sanidad por medio de sulfato de sodio o sulfato de magnesio. CDMX: ONNCCE.

NMX-C-077-ONNCCE. (1997). Industria de la construcción - Agregados para concreto - Análisis granulométrico - Método de prueba. CDMX: ONNCCE.

NMX-C-083-ONNCCE. (2014). Industria de la Construcción-Determinación de la resistencia a compresión. México: Organismo Nacional de Normalización y Certificación de la Construcción y Edificación, S. C.

NMX-C-084-ONNCCE. (2006). Industria de la construcción - Agregados para concreto - Partículas más finas que la criba 0,075 mm (No. 200) por medio de lavado - Método de prueba. CDMX: ONNCCE.

NMX-C-089-ONNCCE. (1997). Industria de la Construcción - Concreto - Determinación de las frecuencias fundamentales, transversal, longitudinal y torcional de especimenesde concreto. CDMX: ONNCCE.

NMX-C-111-ONNCCE. (2014). Industria de la construcción-Agregados para concreto hidráulico-. Especificaciones y métodos de prueba. CMDX: ONNCCE.

NMX-C-122-ONNCCE. (2014). Industria de la Construcción -Agua para Concreto- Especificaciones. México: Organismo Nacional de Normalización y Certificación de la Construcción y Edificación.

NMX-C-122-ONNCCE. (2004). Industria de la Construcción -Agua para Concreto- Especificaciones. CDMX: ONNCCE.

NMX-C-144-ONNCCE. (2010). Industria de la construcción - Cementantes hidráulicos - Requisitos para el aparato usado en la determinación de la fluidez de morteros. CDMX: ONNCCE.

NMX-C-148-ONNCCE. (2010). Industria de la Construcción - Cementos y Concretos Hidráulicos - Gabinetes, Cuartos Húmedos y Tanques de Almacenamiento - Condiciones de Diseño y Operación. México: Organismo Nacional de Normalización y Certificación de la Construcción y Edificación, S.C.

NMX-C-148-ONNCCE. (2010). Industria de la Construcción - Cementos y Concretos Hidráulicos - Gabinetes, Cuartos Húmedos y Tanques de Almacenamiento - Condiciones de Diseño y Operación. CMDX: ONNCCE. 
NMX-C-152-ONNCCE. (2010). ndustria de la construcción - Cemento hidráulico - Determinación de la densidad. CDMX: ONNCCE.

NMX-C-159-ONNCCE. (2004). Industria de la Construcción - Concreto - Elaboración y Curado de Especímenes en el Laboratorio. México: Organismo Nacional de Normalización y Certificación de la Construcción y Edificación, S.C.

NMX-C-159-ONNCCE. (2004). Industria de la Construcción - Concreto - Elaboración y Curado de Especímenes en el Laboratorio. CDMX: ONNCCE.

NMX-C-160-ONNCCE. (2004). Industria de la construcción - Concreto - Elaboración y curado en obra de especímenes de concreto. CDMX: ONNCCE.

NMX-C-163-ONNCCE. (1997). Industria de la construcción - Concreto - Determinación de la resistencia a la tensión por compresión diametral de cilindros de concreto. CDMX: ONNCCE.

NMX-C-165-ONNCCE. (2014). Industria de la Construcción - Agregados - Determinación de la Masa Específica y Absorción de Agua del Agregado Fino - Método de Prueba. México: Organismo Nacional de Normalización y Certificació de la Construcción y Edificación, S.C.

NMX-C-165-ONNCCE. (2014). Industria de la construcción - Agregados - Determinación de la densidad relativa y absorción de agua del agregado fino - Método de ensayo. CDMX: ONNCCE.

NMX-C-166-ONNCCE. (2006). Industria de la Construcción - Agregados - Contenido de Agua por Secado Método de Prueba. CDMX: ONNCCE.

NMX-C-170-ONNCCE. (1997). Industria de la Construcción - Agregados - Reducción de las Muestras de Agregados Obtenidas en el Campo, al Tamaño Requerido para las Pruebas. CDMX: ONNCCE.

NMX-C-177-ONNCCE. (1997). Industria de la construcción - Concreto - Determinación del tiempo de fraguado de mezclas de concreto, mediante la resistencia a la penetración. CDMX: ONNCCE.

NMX-C-191-ONNCCE. (2015). Industria de la Construcción - Concreto - Determinación de la Resistencia a la Flexión del Concreto Usando una Viga Simple con Carga en los Tercios del Claro. México: Organismo Nacional de Normalización y Certificación de la Construcción y Edificación S.C.

NMX-C-191-ONNCCE. (2015). Industria de la Construcción - Concreto - Determinación de la Resistencia a la Flexión del Concreto Usando una Viga Simple con Carga en los Tercios del Claro. CDMX: ONNCCE.

NMX-C-205-ONNCCE. (2005). Industria de la construcción - Concreto - Determinación de la resistencia del concreto a la congelación y deshielo acelerados. CDMX: ONNCCE.

NMX-C-251-ONNCCE. (1997). Industria de la construcción - Concreto - Terminología. CDMX: ONNCCE.

NMX-C-263-ONNCCE. (2010). Industria de la Construcción. CDMX: ONNCCE.

NMX-C-303-ONNCCE. (2010). Industria de la Construcción-concreto hidráulico - determinación de la resistencia a la flexión usando una viga simple con carga en el centro del claro. CDMX: 2010.

NMX-C-403-ONNCCE. (1999). Industria de la construcción - concreto- hidráulico para uso estructural. CDMX: ONNCCE.

NMX-C-414-ONNCCE. (2014). Industria de la construcción-cementos hidráulicos-Especificaciones y métodos de prueba. México: Organismo Nacional de Normalización y certificación de la construcción y edificación, S.C. NMX-C-414-ONNCCE. (2014). Industria de la construcción -cementos hidráulicos- Especificaciones y métodos de prueba. CDMX: ONNCCE.

NMX-C-480-ONNCCE. (2014). Industria de la construcción - Geotecnia - Equivalente de arena de agregados finos - Método de ensayo. CDMX: ONNCCE.

NMX-C-514-ONNCCE. (2016). Industria de la construcción - Resistividad eléctrica del concreto hidráulico especificaciones y métodos de ensayo. CDMX: ONNCCE.

ONU. (23 de 06 de 2017). World Population History. Obtenido de http://worldpopulationhistory.org

Payam Shafigh, H. B. (2013). Agricultural wastes as aggregate in concrete mixtures - A review. Malaysia: ELSEVIER.

Pérez, A. (2012). Morteros, pastas y lechadas. Obtenido de FICAL: Foro Ibérico de la cal: http://www.fical.org/index.php?option=com_content\&view=article\&id=57:morteros-pastas-ylechadas\&catid $=42$ : clasificacion $\&$ Itemid $=145$

Phipps, J. (1997). Monograph of northern mexican Crataegus (Rosaceae, sufam. Texas, USA.

Propiedades de los cementos. (2017). Uniovi. Obtenido de http://www6.uniovi.es/usr/fblanco/Leccion6.PropiedadesCEMENTOS.pdf

Ragai. (1987). Surface and bulk properties of ancient egyptian mortars. Part II: adsorption and infrared studies. 18:9-17. 
Ragai, J. (1989). Surface and bulk properties of ancient egyptian mortars. Part V: thermal studies. 19:42-46. Ragai. (1988). Surface and bulk properties of ancient egyptian mortars. Part lll: X-ray diffraction studies. 18:1917.

Ramezanianpour AA, K. M. (2009). The effect of rice husk ash on mechanical properties and durability of sustainable concretes. Int J Civil Eng.

RED DURAR (Durabilidad de la armadura). (2000). Manual de Inspección, Evaluación y Diagnóstico de Corrosión en Estructuras de Hormigón Armado. México: CYTED.

Santarelli, L. (1960). Elementi di chimica del cemento.

Vaca Arciga, L. A. (2017). [Película]. 